\title{
Aminosäuren in der parenteralen Ernährung
}

Früher wurde besonders in der Chirurgie die Ansicht ver-treten, daß es unsinnig sei, in der sogenannten katabolen Phase Substrate zuzuführen, und daß die Nahrungskarenz geradezu eine Entlastung des Organismus darstelle. Die Proteinkatabolie wurde als schicksalhafte Erscheinung auf-gefaßt, weil sie auf eine verminderte Proteinresynthese und nicht auf einen gesteigerten Proteinabbau zurückge-führt wurde. Inzwischen konnte durch Untersuchungen von Hartig (Abbildung 1) sichergestellt werden, daß die Fähigkeit zur Synthese von Körpereiweiß in Streßsituatio-nen praktisch nicht gestört ist, daß allerdings mit zuneh-mendem Streßgrad die Abbaurate weiter ansteigt. Streß-bedingte Stickstoffverluste können also durch nutritive Maßnahmen zumindest deutlich vermindert werden. Auf-grund dieser Erkenntnisse geht es nicht an, daß Patienten in einer Phase äußerster Belastung einer Nulldiät mit Was-ser und Elektrolyten unterzogen werden. Vielmehr sollten zusätzlich ausreichende Mengen geeigneter Kalorienträ-ger (siehe auch "pfrimmer informiert « Klinikarzt Heft 7, 1975) und Aminosauren in bedarfsadaptierten Proportio-nen im Rahmen parenteraler Ërnährung appliziert werden. Beim Studium der heute vorliegenden Literatur zur parenteralen Aminosäurensubstitution kristallisieren sich drei Arbeitsgruppen in der BRD heraus, die sich eingehend mit der Frage einer optimalen Relation in der Zufuhr der essentiellen Aminosauren beschäftigt haben. Ausgehend von verschiedenen theoretischen Ansätzen, stimmen die von diesen Arbeitsgruppen bei Versuchspersonen und Pa-tientenkollektiven erarbeiteten Aminosäurenmuster hin-sichtlich der Proportionierung der klassischen essentiellen Aminosauren mit Ausnahme des Bedarfs an schwefelhal-tigen Aminosauren in einem Bereich von \pm 10 bis 15\% überein. Von Dolif/JüRGENS wird vorgeschlagen, daß dieses Pattern als Normmuster mit hohem und niedrigem Ge-halt an schwefelhaltigen Aminosauren den verbindlichen Rahmen für die Proportionierung in Infusionslösungen dar-stellen sollte. Die Normmuster mit niedrigem Gehalt an schwefelhaltigen Aminosauren wurden vorwiegend bei parenteraler Ërnährung im Minimalbereich entwickelt, das Normmuster mit hohem Gehalt an schwefelhaltigen Aminosauren dagegen bei mittlerer bis hoher parenteraler Aminosäurenzufuhr. In Übereinstimmung damit konnte Hegsted zeigen, daß bei höherer Stickstoffzufuhr, das heißt beim Bemühen, eine Bilanz zu positivieren, der relative Bedarf an der schwefelhaltigen Aminosäure Methio-nin überproportional ansteigt, im Beispiel der Tabelle I áxxî

367

493 \%. Es zeigt sich dabei, daß nicht die absolute Zufuhr einer Aminosäure entscheidend ist, sondern vielmehr ihr Verhältnis zu den weiteren zugeführten Aminosauren. Für eine in der Klinik im allgemeinen erforderlichen Ërnährung mit 0,8 bis 1,6 g Aminosauren pro Kilogramm Kör-pergewicht und Tag ist ein Normmuster mit hohem rela-tiven Gehalt an schwefelhaltigen Aminosauren notwendig. Entsprechend den Untersuchungsergebnissen von Dolif/ Jürgens ist die seit Rose vorgenommene strenge Tren- 
Tabelle II. Infusionsplan: 3000 ml Flüssigkeit, 2100 Kalorien, 75 g Aminosäuren, 450 g

Kohlenhydrate.

Aminosäuren Fructose

$$
\text { g g }
$$

$500 \mathrm{ml}$ Triofusin E $1000 \quad 60$

$500 \mathrm{ml}$ Aminofusin L $600 \quad 25$

$500 \mathrm{ml}$ Triofusin E $1000 \quad 60$

$500 \mathrm{ml}$ Aminofusin L $600 \quad 25$

$500 \mathrm{ml}$ Triofusin E $500 \quad 30$

500 ml Aminofusin L $600 \quad 25$

$3000 \mathrm{ml}$ Infusionslösungen $75 \quad 150$

ung der Bausteinaminosäuren in 8 essentielle und im übrigen nichtessentielle Aminosäuren als

nicht mehr zweckmäßig anzusehen. Untersuchungen von Bale-strieri weisen darauf hin, daß im

Rahmen parenteraler Ernährung lediglich 5 Bausteinaminosäuren in ausreichen-der Menge

endogen synthetisiert werden. Dies sind in ab-steigender Reihenfolge Asparaginsäure,

Glutaminsäure, Serin, Alanin und Glycin. Die nach der klassischen Ein-teilung als nichtessentiell

bezeichneten Aminosäuren Ar-ginin, Histidin, Prolin und Alanin haben sich aufgrund von

parenteralen Ernährungsstudien der letzten Jahre als mehr oder weniger essentiell herausgestellt.

Sie sollten in bestimmten Proportionen zusätzlich zu den 8 klassischen essentiellen Aminosäuren

verabreicht werden. Entspre-chend Dolif/Jürgens muß der Proportionierung der nichtessentiellen

Aminosäuren die gleiche Aufmerksam-keit geschenkt werden wie bei den essentiellen

Aminosäuren. Glycin als einzige unspezifische Stickstoffquelle führt selbst bei optimaler

Zusammensetzung des essentiellen Stickstoffs zu stark negativen Stickstoffbilanzen. Es ware jedoch verfehlt, hieraus die Schlußfolgerung zu zie-hen, auf Glycin als nichtessentielle

Stickstoffquelle zu verzichten. Glycin ist unerläßlich für die Bildung von Hip-pursäure,

Glutathion, Kreatin, konjungierten Gallensäu-ren, Porphyrinen und Purinen. Die beiden letzten

sind die Voraussetzungen für Hämatopoese und Nucleinsäuresyn-these. Abbildung 2 zeigt, daß

Glycin- und Serinspiegel bei Infusionen von Aminofusin ${ }^{\circledR}$ nach Einstellung eines steady state im schraffierten Normbereich liegen. Dieses Ergebnis ist nur durch kombinierte Zufuhr anderer nichtessentieller Aminosäuren zu erzielen. In diesem Zusammenhang soil darauf hingewiesen werden, daß die Glutaminsäure eine ideale Quelle nichtessentiellen Stickstoffs darstellt, weil sie besonders leicht Transaminierungsreaktionen eingeht.

Serum

mg/100ml Glycin Serin

Abbildung 2. Serumkonzentration von Glycin und Serin bei Infusion von Aminofusin nach

Einstellung eines steady state (nach

DOLIF/JÜRGENS).

Literatur

Balestrieri, C.; Cittadini, D., and Giordano, C: Essential and non-essential amino acids in 15H

and 14C labeling studies in normal and in uremic subjects. Life Sci. 7:1033-1038 (1968).

Dolif, D. und Jürgens, P.: Bedarf und Verwertung der Aminosäuren; in Ahnefeld, Burri, Dick und Halmágyi Infusions-therapie II, Parenterale Ernährung. Klinische Anästhesiologie und Intensivtherapie, vol. 7 (Springer, Berlin 1975).

Dolif, D. und Jürgens, P.: Utilisation von Aminosäuren bei parenteraler Applikation; in Lang, Fekl und Berg Bilanzierte Ernährung in der Therapie (Thieme, Stuttgart 1971). 
Hartig, W.; Wetzel, K.; Gebhardt, O.; Czarnetzki, H.-D. und Hübner, G: Der postoperative Eiweißstoffwechsel. 2. Mitt: Die Änderung des Eiweißstoffwechsels durch eine Operation sowie die Verwertung postoperativ zugeführten Stickstoffs (Untersuchungen an Ratten mit markiertem Stickstoff). Z. exper. Chir. 5:56 (1970).

Hegsted, D. M.: Variation in requirements of nutrients amino acid. Fed. Proc. Fed. Am. Socs. 